\title{
Visual function assessment of posterior-chamber phakic implantable collamer lenses with a central port
}

\author{
Zongxia Bai^, Danyao Nie, Jing Zhang, Huiling Hu, Liangnan Sun, Kun Zeng, Jiantao Wang, Xinhua Liu \\ The Second Clinical Medical College, Jinan University (Shenzhen Eye Hospital), Shenzhen, China \\ Contributions: (I) Conception and design: Z Bai; (II) Administrative support: X Liu, J Wang; (III) Provision of study materials or patients: X Liu; (IV) \\ Collection and assembly of data: Z Bai, J Zhang; (V) Data analysis and interpretation: Z Bai, D Nie, H Hu; (VI) Manuscript writing: All authors; (VII) \\ Final approval of manuscript: All authors. \\ Correspondence to: Xinhua Liu. Shenzhen Eye Hospital,18\# Zetian Road, Shenzhen 518000, China. Email: xhualiu@sohu.com.
}

\begin{abstract}
Background: To investigate the short-term visual function of the V4c implantable collamer lens (ICL) for myopia using a Binoptometer 4P.

Methods: Eighty eyes from 40 patients (age, 28.75 \pm 6.57 years) who underwent ICL V4c implantation at Shenzhen Eye Hospital from August 2020 to June 2021 were enrolled. Uncorrected visual acuity (UCVA), best corrected visual acuity (BCVA), subjective manifest refraction, intraocular pressure (IOP), and corneal endothelial cell density (ECD) were evaluated 1 month after surgery. Corrected distance visual acuity (CDVA), corrected near visual acuity (CNVA), contrast sensitivity (CS), near stereoacuity (NSA), twilight vision, and glare sensitivity were measured using a Binoptometer 4P.

Results: The average logMAR (logarithmic minimum angle of resolution) UCVA postoperative was $0.07 \pm 0.13$, which was significantly better than the preoperative BCVA $(\mathrm{P}=0.028)$. At 1 month postoperatively, the mean safety and efficacy indexes were $1.14 \pm 0.24$ and $0.98 \pm 0.16$, respectively. The mean spherical equivalent (SE) of all eyes was $-8.98 \pm 3.20 \mathrm{D}$ preoperatively, and $-0.16 \pm 0.48 \mathrm{D}$ postoperatively. None of the patients lost one or more lines of BCVA, $62.1 \%$ remained unchanged, $10.6 \%$ gained one line, and $27.3 \%$ gained two or more lines. CDVA, CNVA, CS, and NSA of both eyes were measured using a Binoptometer $4 \mathrm{P}$, which were significantly better than the preoperative values. In addition, some patients had subjective symptoms in the early postoperative period, such as halo, glare, etc.

Conclusions: ICL V4c implantation is a safe, effective, and predictable solution for myopia. We found that the short-term visual function of patients was improved significantly. The Binoptometer $4 \mathrm{P}$ is an effective and convenient visual testing device for assessing postoperative visual function after ICL V4c implantation.
\end{abstract}

Keywords: Myopia; phakic intraocular lens; V4c; visual function; Binoptometer 4P

Submitted Nov 25, 2021. Accepted for publication Feb 16, 2022.

doi: $10.21037 /$ atm-22-107

View this article at: https://dx.doi.org/10.21037/atm-22-107

\section{Introduction}

Laser corneal refractive surgery for myopia correction has been widely accepted. However, this involves a risk of iatrogenic keratoconus in patients with moderate to high refractive errors or low cornea thickness. The implantable collamer lens (ICL; STAAR Surgical, Nidau, Switzerland) is a posterior-chamber phakic lens for refractive correction. The commercially available model of this lens (V4c) since 2011 incorporates a central port of $0.36 \mathrm{~mm}$ (KSAquaport). The port allows physiologic flow of aqueous without the need for Nd:YAG peripheral iridotomies or intraoperative iridectomies. Compared with corneal laser

^ ORCID: 0000-0001-7407-2574. 
surgery, concerns related to ICL safety have included lens exchange or explantation, pupillary block, endothelial cell loss, pigment dispersion, elevated intraocular pressure (IOP) and cataract. However, long-term outcomes confirmed that ICL V4c implantation provided good results in terms of safety, efficacy, predictability, and stability (1). The ICL V4c corrects -0.50 to $-18.00 \mathrm{D}$ myopic spherical refraction and up to $-5.00 \mathrm{D}$ cylindrical refraction. Posterior-chamber phakic implantable collamer lenses V4c (ICL V4c) has the advantages of reversibility and rapid recovery, as well as retaining the integrity of the cornea and the adjustment function of the lens. Previous studies have shown that ICL V4c implantation has better objective visual effects in contrast sensitivity and high-order aberration (HOAs) than laser refractive surgery $(2,3)$.

Previous instruments used to evaluate visual quality after ICL V4c implantation included the double-pass optical quality analysis system (OQAS II), CSV-1000 multifunctional visual acuity instrument, stereoscopic atlas, etc. However, a comprehensive assessment of visionrelated competence should consider both visual function (the performance of various components of the visual system) and functional vision (the ability to perform visual tasks). Typical visual function assessments include visual acuity, contrast sensitivity (CS), color, depth, and motion perception, which respectively represent one aspect of visual function and may affect individuals' functional vision level (4). The Binoptometer $4 \mathrm{P}$ is a vision test device, which can be used to determine visual acuity, test binocular vision, color sensitivity, contrast sensitivity, and peripheral visual field perception. You also have the option to test twilight vision and glare sensitivity. Using a binoptometer 4P, we can perform a quantitative analysis of some indicators of visual function to more specifically describe the alterations of visual function in patients. In this study, a Binoptometer $4 \mathrm{P}$ was used to test the far $(5 \mathrm{~m})$ and near $(40 \mathrm{~cm})$ vision, $\mathrm{CS}$, near stereoacuity (NSA), twilight vision, and glare sensitivity of patients with monocular and binocular vision. It can be used to conveniently observe the early postoperative comprehensive visual function of ICL.

We present the following article in accordance with the STROBE reporting checklist (available at https://atm. amegroups.com/article/view/10.21037/atm-22-107/rc)

\section{Methods}

Eighty eyes from 40 patients (age, $28.75 \pm 6.57$ years) who underwent ICL V4c implantation at Shenzhen Eye Hospital from August 2020 to June 2021 were enrolled. The inclusion criteria were as follows: (I) age $\geq 18$ years; (II) stable refractive error $(\leq 0.50 \mathrm{D}$ change per year in refractive error in the past 2 years); and (III) minimum anterior chamber depth (ACD) $\geq 2.8 \mathrm{~mm}, \mathrm{ECD} \geq 2,000$ cells $/ \mathrm{mm}^{2}$. The exclusion criteria were as follows: (I) patients with uveitis, keratoconus, glaucoma, cataract, retinitis pigmentosa, and other eye diseases; (II) patients with a history of ocular trauma or surgery; and (III) patients with systemic diseases, such as hyperthyroidism and autoimmune diseases, or severe mental disorders, such as anxiety and depression. All procedures performed in this study involving human participants were in accordance with the Declaration of Helsinki (as revised in 2013). The study was approved by the ethics board of Shenzhen Eye Hospital (No. b-07) and informed consent was taken from all the patients.

\section{Preoperative examination}

Before ICL V4c implantation, patients underwent complete ophthalmologic examination, including uncorrected visual acuity (UCVA), best corrected visual acuity (BCVA), manifest and cycloplegic refractions, slit lamp microscope and fundoscopic examination, corneal topography (Oculus, Germany), ACD measurement (SW-3200L; Chasowa, China, Tianjin), corneal endothelial cell density (ECD) measurement (SP 3000P; Topcon, Janpn), intraocular pressure (IOP) measurement using a non-contact tonometer (NCT; Canon TX-20, Japan), and Binoptometer 4P (Oculus, Germany).

\section{Surgical technique}

ICL V4c implantation procedures were performed by the same experienced surgeon. Preoperatively, levofloxacin (Cravit; Santen, Osaka, Japan) eye drops were applied topically four times daily for 3 days. The pupils were fully dilated (Tropicamide Phenylephrine Eye Drops) and surface anesthesia (Proparacaine Hydrochloride Eye Drops) was performed before surgery. A $3.0 \mathrm{~mm}$ corneal incision was made at the temporal side of the eye, and the ICL was inserted into the anterior chamber. After the ICL had unfolded naturally, a viscoelastic agent was injected to maintain the appropriate anterior chamber depth. The ICL loop was placed into the posterior ciliary sulcus of the iris (with a positioning hook) and in the center of the ICL optical area. Subsequently, the viscoelastic surgical agent was completely removed using a balanced salt solution. Eye 
ointment was applied to the conjunctival sac and the eye was covered after the operation. After surgery, the patients were given levofloxacin eye drops four times daily for 1 week.

\section{Postoperative examination}

Routine follow-up was performed on all patients at 1 day, 1 week and 1 month postoperatively. At 1-month postoperatively, the following parameters were measured: UCVA, BCVA, IOP, and subjective manifest refraction. CDVA, CNVA, CS, NSA, twilight vision, and glare sensitivity were measured using the Binoptometer 4P.

\section{Binoptometer $4 P$}

\section{Visual acuity tests with tumbling $\mathbf{E}$ charts}

Five E letters are generally displayed per visual acuity level, of which at least three must be identified in order to pass that visual acuity level. Using this test, visual acuity testing can be performed for different distances and visual acuity levels. In this study, the visual acuity of the patients was measured from far $(5 \mathrm{~m})$ and near $(40 \mathrm{~cm})$.

\section{Contrast sensitivity}

The contrast of E letters can be changed to examine the patients' CS.Testing of the CS is done under normal daylight conditions (photopic), as is the visual acuity test. The $\mathrm{E}$ letter with a bright background are presented to the patient. While high contrast $\mathrm{E}$ letter are presented for visual acuity testing, thecontrast of the E letter can be gradually reduced for this test. The adopted criterion was to correctly identify at least three out of the five visual targets (5).

\section{Stereo vision tests with crosses}

This involves the patient seeing five lines, each with five crosses in the device. In each line, one cross stands out from the other crosses in that line. Various different stereo threshold angles were tested in the first stereo test. At the end of the examination, we could assess whether stereoscopic vision is present as well as the quality of the stereovision. Grade detection was performed according to the parallax gradient of 600 arcsecond, 400 arcsecond, 200 arcsecond, 100 arcsecond, 80 arcsecond, 60 arcsecond, 45 arcsecond, 30 arcsecond, and 15 arcsecond until the protruding crosses could not be accurately identified, and the results were recorded. If the cross mark with the greatest parallax gradient could not be identified, the result was recorded as "no stereopsis".

\section{Twilight vision and glare sensitivity}

Twilight (mesopic) vision is defined as the vision range between daylight vision and night vision. To adapt the test conditions to the luminance conditions prevailing on the road at night, the optotype character was presented on a test field with a brightness of $0.032 \mathrm{CD} / \mathrm{m}^{2}$, and to test with glare, a test field with a brightness of $0.1 \mathrm{CD} / \mathrm{m}^{2}$ was used. Unlike the visual acuity test, the size of the optotype character was not reduced in this test, but rather its contrast with respect to its surroundings. The size of the Landolt rings corresponded to a visual acuity of 20/200. Four different contrast levels (1:23, $1: 5,1: 2.7,1: 2)$ are available for diminishing the contrast, and a contrast level of 1:23 represents the greatest contrast, which is the easiest to identify. A contrast of 1:23 signifies the ratio of the luminosity of the optotype character to the luminance of the surrounding area.

\section{Evaluation criteria}

For evaluation of the common curative effect, the efficacy index represented the ratio between the UCVA at 1 month and the corresponding preoperative BCVA, and the safety index was calculated as the ratio between the BCVA at 1 month and the corresponding preoperative BCVA. For evaluation of visual function, the stereopsis gradients in Binoptometer 4P included 600 arcsecond, 400 arcsecond, 200 arcsecond, 100 arcsecond, 80 arcsecond, 60 arcsecond, 45 arcsecond, 30 arcsecond and 15 arcsecond. The recorded results were divided into three grades: good ( $\leq 60$ arcsecond), medium (>60 arcsecond and $\leq 200$ arcsecond), and poor (>200 arcsecond or no stereoscopic vision) (6). With fixed scopes, the lower the contrast level, the better the visual function. A study by the University of Tubingen in Germany recommended a standard value of 0.4 for the recognition of a comparison level of $15 \%$. Also, the normal reference value of intermediate vision is $\leq 1: 2.7$, and the normal reference value of glare sensitivity is $\leq 1: 2.7$ (5).

\section{Statistical analysis}

Statistical analysis was performed using SPSS for Windows (23.0, SPSS Inc., Chicago, Illinois, SAD) and Microsoft Excel (11.0, Microsoft Corporation, Redmond, WA, SAD). Quantitative data were expressed by mean \pm standard deviation (mean $\pm \mathrm{SD}$ ). Visual acuity was converted to logarithmic minimum angle of resolution (LogMAR) visual acuity for statistical analysis. The KolmogorovSmirnov test was used to determine whether a variable was 
Table 1 Preoperative patient demographics

\begin{tabular}{lc}
\hline Characteristic & Mean \pm SD (range) \\
\hline Age (years) & $28.75 \pm 6.57[19-47]$ \\
Gender (male: female) & $8: 32$ \\
Manifest spherical equivalent (D) & $-8.98 \pm 3.20(-3.00$ to -15.50$)$ \\
Manifest cylinder (D) & $-1.30 \pm 1.06(-4.50-0)$ \\
LogMAR UCVA & $1.32 \pm 0.31(0.92-2.00)$ \\
LogMAR BCVA & $0.06 \pm 0.13(0.00-0.80)$ \\
White-to-white distance (mm) & $11.51 \pm 0.40(10.80-12.50)$ \\
Anterior chamber depth (mm) & $3.11 \pm 0.20(2.80-3.48)$ \\
Endothelial cell density & $2,862.52 \pm 322.66$ \\
(cells/mm²) & $(2,051.3-3,972.6)$ \\
\hline
\end{tabular}

$\mathrm{SD}$, standard deviation; D, diopter; LogMAR, logarithmic minimum angle of resolution; UCVA, uncorrected visual acuity; BCVA, best corrected visual acuity.

normally distributed, and the paired sample $t$ test was used if the distribution is normal. The comparison between the preoperative and postoperative periods was performed with the Wilcoxon signed rank test. Grade data were compared using the $\chi^{2}$ test. $\mathrm{P}<0.05$ was considered statistically significant.

\section{Results}

\section{Demographic information}

The preoperative demographics of the included patients are summarized in Table 1. LogMAR UCVA and BCVA were $1.32 \pm 0.31$ (range, $0.92-2.00$ ) and $0.06 \pm 0.13$ (range, $0.80-0.00)$, respectively. The horizontal white-to-white distance was $11.51 \pm 0.40 \mathrm{~mm}$ (range, $10.80-12.50 \mathrm{~mm}$ ) and the anterior chamber depth was $3.11 \pm 0.20 \mathrm{~mm}$ (range, $2.80-3.48 \mathrm{~mm}$ ). The intraocular pressure (IOP) was $13.98 \pm 2.34 \mathrm{mmHg}$ (range, $11-14 \mathrm{mmHg}$ ) and the endothelial cell density was $2,862.52 \pm 322.66$ cells $/ \mathrm{mm}^{2}$ (range, 2,051.3-3,972.6 cells $/ \mathrm{mm}^{2}$ ).

\section{Efficacy}

The mean postoperative UCVA at 1 month $(0.07 \pm 0.13$ $\log$ MAR) was statistically significantly better than the preoperative BCVA $(\mathrm{P}=0.028$, Wilcoxon signed-rank test). The efficacy index (defined as BCVA at 1 month/BCVA preoperatively) was $0.98 \pm 0.16$. A total of 80 eyes $(100 \%)$ gained $\geq 2$ lines of UCVA. Also, $97.5 \%$ eyes had a decimal
UCVA of $0.5(20 / 40)$ or better at the follow-up visit. One month after surgery, $87.2 \%$ and $60.3 \%$ of eyes had a UCVA of $20 / 25$, and of $20 / 20$ or better, respectively.

\section{Safety}

The mean BCVA at 1 -month postoperatively was $0.00 \pm 0.06$ $\log M A R$. There was a significant difference between preoperative BCVA and 1-month postoperative BCVA $(\mathrm{P}<0.05$, Wilcoxon signed-rank test). The safety index (defined as UCVA at 1-month postoperatively/BCVA preoperatively) was $1.14 \pm 0.24$. None of the subjects had a loss of BCVA after surgery, 27.3\% (18/66) had an increase of two lines or more after surgery, 10.6\% (7/66) had an increase of one line, and $62.1 \%$ (41/66) were unchanged.

\section{Predictability}

Manifest SE was significantly decreased from $-8.98 \pm 3.20 \mathrm{D}$ preoperatively to $-0.16 \pm 0.48 \mathrm{D}$ at 1 -month postoperatively $(\mathrm{P}<0.001$, Wilcoxon signed-rank test $)$. Also, at 1 -month after surgery, 69 eyes $(86.6 \%)$ were within $\pm 0.5 \mathrm{D}$ of the attempted SE, and 78 eyes (97.5\%) were within $\pm 1.0 \mathrm{D}$.

\section{Intraocular pressure}

Preoperatively, the mean IOP was $13.98 \pm 2.34 \mathrm{mmHg}$. At 1 -month postoperatively, the mean IOP was $14.30 \pm 2.90 \mathrm{mmHg}$. No significant increase in IOP $(>21 \mathrm{mmHg})$ occurred in any case during the 1-month follow-up.

\section{Monocular/binocular CDVA, CNVA}

The monocular LogMAR CDVA and CNVA changed significantly from $0.10 \pm 0.16$ and $0.11 \pm 0.18$ preoperatively to $0.02 \pm 0.10$ and $0.06 \pm 0.12$ at 1 -month postoperatively, respectively ( $\mathrm{P}<0.001$, Wilcoxon signed-rank test). Also, the binocular LogMAR CDVA and CNVA changed significantly from $0.05 \pm 0.10$ and $0.06 \pm 0.12$ preoperatively to $-0.01 \pm 0.08$ and $0.01 \pm 0.06$ at 1 -month postoperatively, respectively $(\mathrm{P}<0.001$, Wilcoxon signed-rank test), as shown in Table 2 and Figure $1 A$.

\section{Contrast sensitivity}

The chi-square test was used according to two grades of normal reference value 0.4 and $3 \mathrm{~m}$; CS $\leq 15 \%$ was normal, while CS $>15 \%$ was abnormal. The results showed that 
Table 2 Comparison of far and near visual acuity in one or both eyes before and after surgery in patients with ametropia

\begin{tabular}{lccc}
\hline Vision & Mean \pm SD (preop) & Mean \pm SD (postop 1m) & P value \\
\hline Monocular CDVA $(5 \mathrm{~m})$ & $0.10 \pm 0.16$ & $0.02 \pm 0.10$ & 0.000 \\
Monocular CNVA $(40 \mathrm{~cm})$ & $0.11 \pm 0.18$ & $0.06 \pm 0.12$ & 0.000 \\
Binocular CDVA $(5 \mathrm{~m})$ & $0.05 \pm 0.10$ & $-0.01 \pm 0.08$ & 0.000 \\
Binocular CNVA $(40 \mathrm{~cm})$ & $0.06 \pm 0.12$ & $0.01 \pm 0.06$ & 0.002
\end{tabular}

Values < 0.05 were considered statistically significant; SD, standard deviation; Preop, preoperative visit; Postop $1 \mathrm{~m}$, 1 month follow-up; CDVA, corrected distance visual acuity; CNVA, corrected near visual acuity.

the CS level of both eyes had been significantly improved after surgery compared with that before surgery, and the difference was statistically significant $\left(\chi^{2}=13.244, \mathrm{P}=0.000\right.$; $\left.\chi^{2}=10.313, \mathrm{P}=0.001\right)$. As shown in Figure $1 B$, the proportion of normal CS increased from $37.5 \%$ in monocular and $55.0 \%$ in binocular to $66.25 \%$ in monocular and $87.50 \%$ in binocular. Figure 1C,1D shows the CS distribution of patients after ICL V4c implantation.

\section{Near stereoacuity}

The stereoscopic vision of the patients before surgery was concentrated in peripheral stereoscopic vision, while postoperative stereoscopic vision was mainly in macular vision, as shown in Figure 1E. The chi-square test was performed according to the three grades of good $(\leq 60$ arcsecond), moderate ( $>60$ arcsecond and $\leq 200$ arcsecond), and poor (>200 arcsecond or no stereovision). The postoperative stereovision was improved compared with that before surgery $\left(\chi^{2}=7.221, \mathrm{P}=0.027\right)$, and the difference was statistically significant, as shown in Tables 3,4.

\section{Twilight vision and glare sensitivity}

Before surgery, 13 cases had mesopic vision and glare sensitivity $\leq 1: 2.7$, accounting for $32.5 \%$. At 1 -month postoperatively, 22 cases had mesopic vision and 10 cases had glare sensitivity $\leq 1: 2.7$, accounting for $55 \%$ and $25 \%$, respectively, as shown in Figure $1 F-1 G$. The postoperative twilight vision level was improved compared with that before surgery, and the difference was statistically significant $\left(\chi^{2}=4.11, \mathrm{P}=0.043\right)$; however, there was no statistical difference in glare sensitivity compared with that before surgery $\left(\chi^{2}=0.55, \mathrm{P}>0.05\right)$.

\section{Complications}

There were no cases of intraoperative complications, such as lens injury or suspensory ligament rupture. None of the patients had posterior capsular opacity, pupil block, cataract, corneal endothelial decompensation, endophthalmitis, or other serious complications during the 1-month postoperative follow-up period.

\section{Discussion}

The present study showed that ICL V4c implantation was a highly effective, safe, and predictable option in treating myopic patients, which was consistent with previous reports (7-10). The visual quality of patients after ICL V4c implantation is a concern at present. In a 3-month prospective study, Miao et al. found that patients could obtain high and stable postoperative optical quality soon after surgery (11). Visual quality assessment can be divided into subjective and objective categories, among which subjective visual quality assessment includes visual acuity, contrast sensitivity, glare sensitivity, stereovision, questionnaire, etc. (4). The most commonly used tests for objective evaluation include wavefront aberration and optical quality analysis system II (OQAS II). Numerous researchers have used OQAS II to observe the visual quality after refractive surgery, and the results have demonstrated that the optical quality after ICL V4c implantation is high (12) and has certain stability (11). The Binoptometer $4 \mathrm{P}$ offers a number of visual function examination options, which are made up of different detection items, such as visual acuity, CS, stereo vision, twilight vision and glare sensitivity. The detection method has unified background light, is not affected by the detection light environment, and detect visual functions at different distances according to the detection requirements. It can be more convenient to comprehensively observe visual function of the patient. The Binoptometer 4P establishes a unified standard visual standard, light (no glare $0.032 \mathrm{CD} / \mathrm{m}^{2}$, glare condition $0.1 \mathrm{CD} / \mathrm{m}^{2}$ ) to test monocular or binocular subjective visual function, so as to reduce the error caused by different 

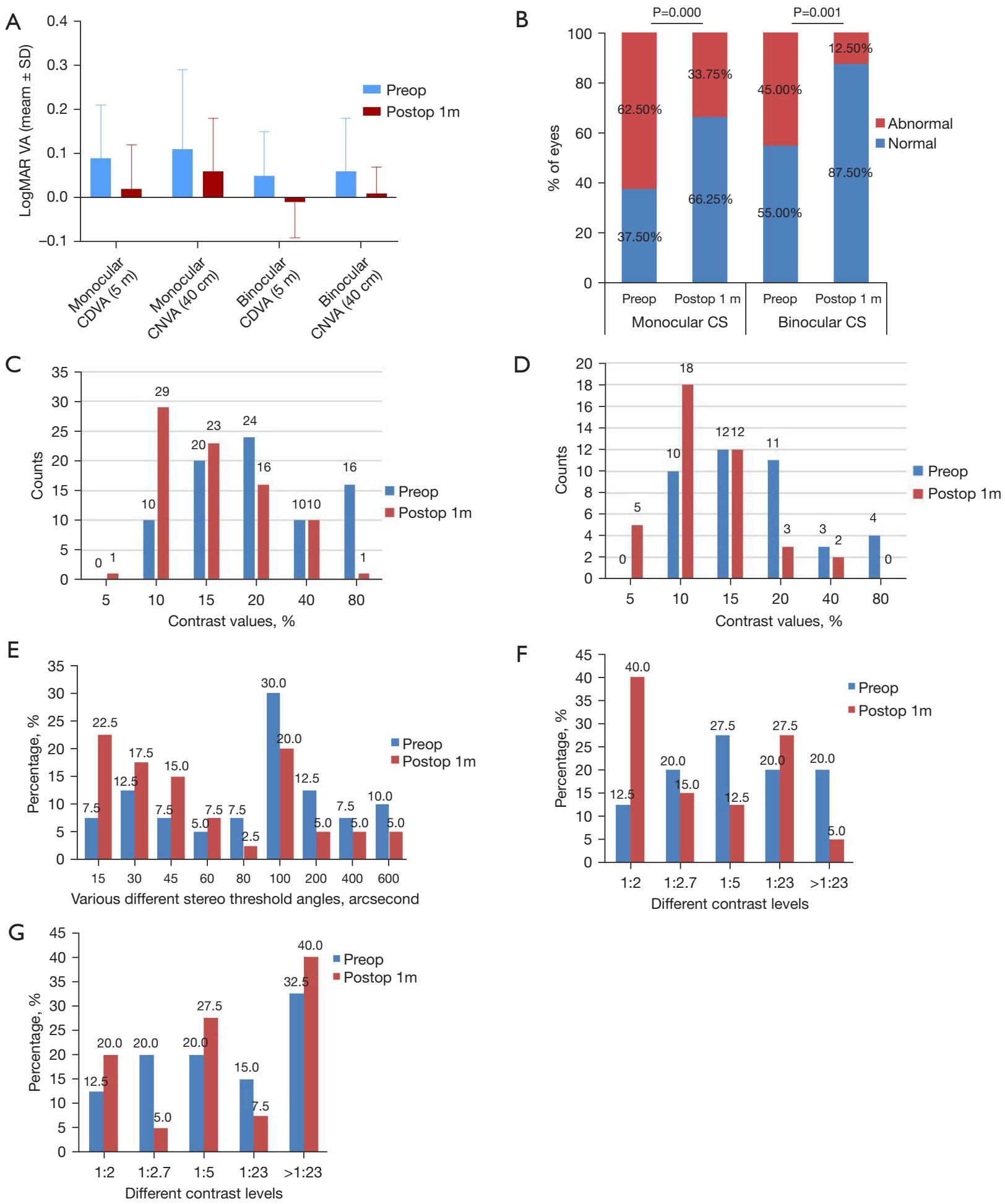

Figure 1 Visual outcomes at 1 month postoperatively in 40 patients with ametropia after implantation of posterior chamber phakic implantable collamer lens (ICL V4c) with a central hole, including (A) distance and near visual acuity in monocular and binocular eyes, (B) change in monocular and binocular CS, (C) distribution of monocular CS at different contrast values, (D) distribution in binocular CS at different contrast values, (E) proportion of different stereoscopic levels after ICL V4c implantation, (F) distribution of twilight vision level, and (G) distribution of glare sensitivity level. Preop, preoperative; Postop, postoperative; SD, standard deviation; m, month; CS, contrast sensitivity. 
Table 3 Description of the percentile of NSA before and after ICL V4c implantation

\begin{tabular}{lcc}
\hline Quartile & $\begin{array}{c}\text { Preop NSA } \\
\text { (arcsecond) }\end{array}$ & $\begin{array}{c}\text { Postop 1m NSA } \\
\text { (arcsecond) }\end{array}$ \\
\hline Median & 94.67 & 48.33 \\
P25 & 48.00 & 25.31 \\
P75 & 194.12 & 100.00 \\
P95 & 600.00 & 500.00 \\
IQR & 146.12 & 74.96 \\
\hline
\end{tabular}

NSA, near stereoacuity; ICL, implantable collamer lens; Postop $1 \mathrm{~m}, 1$ month follow-up; IQR, interquartile range.

Table 4 Prevalence of fine, moderate, and poor stereopsis at near in patients with ICL V4c implantation

\begin{tabular}{lcc}
\hline Result & Preop & Postop 1 m \\
\hline Good & $13 / 40(32.50 \%)$ & $25 / 40(62.50 \%)$ \\
Moderate & $20 / 40(50.0 \%)$ & $11 / 40(27.50 \%)$ \\
Poor & $7 / 40(17.50 \%)$ & $4 / 40(10.0 \%)$ \\
\hline
\end{tabular}

$\mathrm{ICL}$, implantable collamer lens; Preop, preoperative visit; Postop $1 \mathrm{~m}, 1$ month follow-up.

inspection equipment. In this study, the early visual function after ICL V4c implantation was analyzed from multiple perspectives, such as near and distance vision, CS, and stereoscopic vision.

The Binoptometer 4P visual acuity examination reflected the visual sensitivity at different distances with $100 \%$ contrast. To evaluate subjective visual quality at different distances, the visual acuity of the single and double eyes of patients was examined at $5 \mathrm{~m}$ and $40 \mathrm{~cm}$, respectively. The traditional method for whole-course visual acuity examination is mainly the defocus curve, which needs to be completed under a comprehensive optometer, and has the disadvantages of poor repeatability and low reproducibility, as well as being time consuming $(13,14)$. The visual acuity levels of $5 \mathrm{~m}$ in monocular eye, $40 \mathrm{~cm}$ in monocular eye, $5 \mathrm{~m}$ in binocular eyes, and $40 \mathrm{~cm}$ in binocular eyes measured by Binoptometer $4 \mathrm{P}$ were all improved after surgery, and the differences were statistically significant.

In a study of 201 myopia patients, researchers used stepwise multiple regression analysis to explore the relationship between multiple variables, such as gender, diopter, pupil size, corrected visual acuity, and CS function. They concluded that in the normal population, eyes with lower objective scattering index (OSI) and better CDVA showed higher CS (15). In a study conducted by Zhongyang Sun and Yingping Deng on 25 cases of ICL implantation, it was reported that high-frequency CS was better than preoperative sensitivity at 1 week postoperatively, and the difference was statistically significant. Meanwhile, lowfrequency CS was not statistically different compared with the preoperative sensitivity (16). The femtosecond laser group in bright environment before surgery there was no statistically significant difference of postoperative changes in CS, postoperative in dark environment, CS is reduced, especially in the middle and high spatial frequency band. On the contrary, the CS of the ICL group was improved in spatial frequency bands postoperatively in both bright and dark environments, with statistically significant differences (17). In this study, the CS levels of single and both eyes obtained using a Binoptometer $4 \mathrm{P}$ after surgery were significantly improved compared with those before surgery, and the differences were statistically significant. The reasons for the increase in postoperative CS compared with that before surgery are as follows: (I) the existing refractive errors before surgery were corrected by ICL V4c implantation, thereby effectively improving the existing low-order aberrations before surgery; (II) the position of ICL implantation in the eye was the ciliary sulcus, which is close to the central fovea of macula; (III) after ICL V4c implantation, the retinal amplification effect was increased compared with that of glasses; and (IV) the effect of prism and spherical aberration was eliminated, and the retinal imaging quality was improved.

Stereopsis is the highest form of vision; it is the visual function that can distinguish distance, depth, and height following brain processing of the parallax produced by both eyes. This function is essential for humans to control complex and fine motion behaviors. Stereopsis is based on normal binocular monopsia and binocular fusion ability. With abnormal monocular vision, binocular monopic function, or binocular fusion function, stereopsis may be impaired (18). The existing examination methods include isovision machine, Titmus stereoscopic book, Frisby examination, etc.; however, these are different and cannot simultaneously detect distant and near stereoscopic vision. Researchers compared the stereoscopic results of a binocular functional examination instrument with the commonly used Titmus stereoscopic examination, and the results showed that the binocular functional examination instrument was moderately consistent with the results of the traditional Titmus method, which was a reliable method 
for stereoscopic examination (19). A study on the effect of posterior chamber intraocular lens implantation on the stereopsis of patients with high myopia showed that patients had significantly improved near-far stereopsis after surgery, and peripheral stereopsis accounted for a higher proportion of patients' near-far stereopsis before surgery, while macular stereopsis accounted for a higher proportion after surgery (20). In this study, a Binoptometer $4 \mathrm{P}$ was used to measure the patients' preoperative and postoperative nearstereoscopic acuities. We observed that the postoperative near-stereoscopic acuity was improved compared with that before surgery, and the difference was statistically significant.

Scattering light was an important factor affecting the quality of visual attention. Intraocular scattering light could be formed in the eye screen overlay that caused the eyeball on the retinal image contrast, embodied by the increased scattered light value after refractive surgery. The visual quality of some patients are impaired because of glare and so on (21). Although many myopic patients have good corrected vision, they often complain that their vision is not clear in a dark environment or is blurred by glare (22). It has been shown that glare halo is common in myopia patients, and the size of the glare halo in myopic eyes is not related to the degree of myopia, but is negatively correlated with the CS of the middle and near-middle spatial frequencies (23). Tahzib et al. showed that the primary side effects after refractive surgery were decreased night vision and glare (24). The results of our study showed that the night vision level improved after surgery. Due to the phenomenon of visual field narrowing after wearing frame glasses or corneal contact lenses, problems such as dark adaptation disorder, glare, reduced light sensitivity, and contrast sensitivity will occur, resulting in poor visual quality (25). Our study showed that there was no significant difference in glare sensitivity levels before and after surgery, but in the 1-month postoperative follow-up, the comparison level of some patients did decrease significantly under the interference of light source. The limitation of this study was the small sample size and short follow-up time, which may lead to deviation of the statistical results. However, our findings still have a certain reference value. Our analysis describes few adverse events related to ICL V4c implantation, which support the safety of this lens when implanted for refractive correction. Considering the results of the visual function comprehensively in our study, the visual quality can be improved early in the ICL V4c implantation surgery. The ICL V4c lenses performed well in the treatment of moderate-to-high myopia.

\section{Conclusions}

In this prospective study, ICL V4c implantation was shown to be a safe, effective, and predictable correction of refractive errors. Patients with ICL V4c implantation could obtain high and stable postoperative optical quality and rapid improvement of visual function shortly after surgery.

\section{Acknowledgments}

Funding: This work was supported by the Shenzhen San Ming Project (SZSM201812091), Shenzhen, China.

\section{Footnote}

Reporting Checklist: The authors have completed the STROBE reporting checklist. Available at https://atm. amegroups.com/article/view/10.21037/atm-22-107/rc

Data Sharing Statement: Available at https://atm.amegroups. com/article/view/10.21037/atm-22-107/dss

Conflicts of Interest: All authors have completed the ICMJE uniform disclosure form (available at https://atm. amegroups.com/article/view/10.21037/atm-22-107/coif). The authors have no conflicts of interest to declare.

Ethical Statement: The authors are accountable for all aspects of the work in ensuring that questions related to the accuracy or integrity of any part of the work are appropriately investigated and resolved. All procedures performed in this study involving human participants were in accordance with the Declaration of Helsinki (as revised in 2013). The study was approved by ethics board of Shenzhen Eye Hospital (No. b-07) and informed consent was taken from all the patients.

Open Access Statement: This is an Open Access article distributed in accordance with the Creative Commons Attribution-NonCommercial-NoDerivs 4.0 International License (CC BY-NC-ND 4.0), which permits the noncommercial replication and distribution of the article with the strict proviso that no changes or edits are made and the original work is properly cited (including links to both the formal publication through the relevant DOI and the license). See: https://creativecommons.org/licenses/by-nc-nd/4.0/.

\section{References}

1. Yang $\mathrm{W}$, Zhao J, Zhao J, et al. Changes in anterior lens 
density after Implantable Collamer Lens V4c implantation: a 4-year prospective observational study. Acta Ophthalmol 2021;99:326-33.

2. Chen X, Guo L, Han T, et al. Contralateral eye comparison of the long-term visual quality and stability between implantable collamer lens and laser refractive surgery for myopia. Acta Ophthalmol 2019;97:e471-8.

3. Wei R, Li M, Zhang H, et al. Comparison of objective and subjective visual quality early after implantable collamer lens V4c (ICL V4c) and small incision lenticule extraction (SMILE) for high myopia correction. Acta Ophthalmol 2020;98:e943-50.

4. Bennett CR, Bex PJ, Bauer CM, et al. The Assessment of Visual Function and Functional Vision. Semin Pediatr Neurol 2019;31:30-40.

5. Wilhelm H, Peters T, Durst W, et al. Assessment of mesopic and contrast vision for driving licences: which cutoff values, which methods are appropriate? Klin Monbl Augenheilkd 2013;230:1106-13.

6. Wang JY, Feng XL. A Comparison of Different Stereoacuity Tests. Chinese Journal of Optometry Ophthalmology and Visual Science 2019;21:602-7.

7. Schallhorn S, Tanzer D, Sanders DR, et al. Randomized prospective comparison of visian toric implantable collamer lens and conventional photorefractive keratectomy for moderate to high myopic astigmatism. J Refract Surg 2007;23:853-67.

8. Packer M. The Implantable Collamer Lens with a central port: review of the literature. Clin Ophthalmol 2018;12:2427-38.

9. Montés-Micó R, Ruiz-Mesa R, Rodríguez-Prats JL, et al. Posterior-chamber phakic implantable collamer lenses with a central port: a review. Acta Ophthalmol 2021;99:e288-301.

10. Packer M. Meta-analysis and review: effectiveness, safety, and central port design of the intraocular collamer lens. Clin Ophthalmol 2016;10:1059-77.

11. Miao H, Chen X, Tian M, et al. Refractive outcomes and optical quality after implantation of posterior chamber phakic implantable collamer lens with a central hole (ICL V4c). BMC Ophthalmol 2018;18:141.

12. Huseynova T, Ozaki S, Ishizuka T, et al. Comparative study of 2 types of implantable collamer lenses, 1 with and 1 without a central artificial hole. Am J Ophthalmol 2014;157:1136-43.

13. Dhallu SK, Sheppard AL, Drew T, et al. Factors Influencing Pseudo-Accommodation-The Difference between Subjectively Reported Range of Clear Focus and Objectively Measured Accommodation Range. Vision (Basel) 2019;3:34. 14. McNeely RN, Pazo E, Spence A, et al. Visual quality and performance comparison between 2 refractive rotationally asymmetric multifocal intraocular lenses. J Cataract Refract Surg 2017;43:1020-6.

15. Kamiya K, Shimizu K, Iijima A, et al. Factors influencing contrast sensitivity function in myopic eyes. PLoS One 2014;9:e113562.

16. Sun CS, Deng YP. Visual and refractive outcomes of implantable collamer lens with a central hole for high myopia. International Eye Science 2018;18:963-7.

17. Huang X, Wu GD, Dai ZL. Comparative study of postoperative short-term visual quality of femtosecond laser and implantable collamer lens(ICL) in the treatment of patients with high myopia. China Journal of Modern Medicine 2014;24:101-5.

18. Piano ME, O'Connor AR. The effect of degrading binocular single vision on fine visuomotor skill task performance. Invest Ophthalmol Vis Sci 2013;54:8204-13.

19. Li MD, Wang Y, Zhao XH, et al. Application of binoptometer in stereoscopic screening. Recent Advances in Ophthalmology 2020;40:336-9.

20. Shen D, Ji JW, Liang HC, et al. Effect of posterior chamber phakic intraocular lens implantation for high myopia on the stereopsis. International Eye Science 2016;16:121-3.

21. Barrionuevo PA, Colombo EM, Vilaseca M, et al. Comparison between an objective and a psychophysical method for the evaluation of intraocular light scattering. J Opt Soc Am A Opt Image Sci Vis 2012;29:1293-9.

22. Ieong A, Hau SC, Rubin GS, et al. Quality of life in high myopia before and after implantable Collamer lens implantation. Ophthalmology 2010;117:2295-300.

23. Liu XZ, Xu B, Sun ZY. Study of halo size and contrast sensitivity of myopia by vision monitor. International Eye Science 2019;19:2115-7.

24. Tahzib NG, Bootsma SJ, Eggink FA, et al. Functional outcome and patient satisfaction after Artisan phakic intraocular lens implantation for the correction of myopia. Am J Ophthalmol 2006;142:31-9.

25. Li HW, Li JY, Liu C, et al. Analysis of visual quality after ICL $\mathrm{V} 4 \mathrm{c}$ corrective surgery for high myopia using the double-pass instrument. International Eye Science 2018;18:1353-5.

(English Language Editor: A. Kassem)

Cite this article as: Bai Z, Nie D, Zhang J, Hu H, Sun L, Zeng K, Wang J, Liu X. Visual function assessment of posterior-chamber phakic implantable collamer lenses with a central port. Ann Transl Med 2022;10(4):194. doi: 10.21037/ atm-22-107 